\title{
Raman Spectroscopy as a Tool for Characterisation of Liquid Phase Devices
}

\author{
Ben $\operatorname{Hogan}^{1 *}$, Tatiana Perova ${ }^{2,3}$, Sergey Dyakov ${ }^{4}$, Monica Craciun ${ }^{1}$ and Anna \\ Baldycheva $^{1 \dagger}$. \\ ${ }^{1}$ University of Exeter, College of Engineering Mathematics and Physical Sciences, \\ Exeter, EX4 4QF, UK \\ ${ }^{2}$ Department of Electronic and Electrical Engineering, Trinity College, The University \\ of Dublin, Ireland \\ ${ }^{3}$ ITMO University, 49 Kronverkskiy pr., St.-Petersburg, 197101, Russia \\ ${ }^{4}$ Skolkovo Institute of Science and Technology, Photonics and Quantum Material \\ Center, Nobel street 3, Moscow, Russia \\ *bh341@exeter.ac.uk \\ † a.baldycheva@exeter.ac.uk
}

\begin{abstract}
In this paper, we demonstrate how Raman spectroscopy can be an effective tool for the elucidation of the properties of liquid phase devices, looking at signal enhancement through to beam profiling.
\end{abstract}

The constant demand for greater device functionality combined with device miniaturisation is driving an ever-expanding search for new optoelectronic materials. Dynamically controllable, self-assembling metastructures based on nanoscale objects represent an intriguing paradigm within this search. Such metastructures can be formed by low-dimensional nanoparticles (carbon nanotubes, graphene, transition metal dichalcogenides (TMDCs), metal nanoparticles etc.) dispersed in a fluidic host material. Liquid crystalline properties can be utilised as the driving force of selfassembly; either by using a commercial nematic liquid crystal host fluid, or, through the solvent-induced self-assembly of particles under suitable conditions. The nanocomposites can be readily integrated on silicon chip by means of microfluidic technology, in a CMOS-compatible back-end process, allowing for dynamic control of the dispersed particles through the application of various on-chip stimuli. However, a significant challenge remains in non-destructive characterisation of the materials insitu.

Here, liquid crystalline nanocomposites have been synthesised based on twodimensional (2D) materials including graphene oxide (GO) and TMDCs dispersed in either commercially available liquid crystals or various organic solvents. Here, we present our first results on the formation of metastructures using novel liquid crystalline composites and suitable design of chips or cells to allow dynamic application of aligning fields. We consider how effective chip design can facilitate characterising the resultant structures. Scanning electron microscopy (SEM), atomic force microscopy (AFM) and polarised light microscopy have been applied to image the liquid crystalline composites (Fig. 1). We then show that Raman spectroscopy is a powerful tool for the characterisation of integrated nanocomposites with a high degree of accuracy [1]; from the broad trends in nanoparticle concentration profiles, to the monitoring of the spatial dynamics of individual nanoparticles in three dimensions simultaneously (Fig. 2). Other information that can be extracted includes; shapes, sizes and aspect ratios of particles and information about the material 
quality and defects. We determine how different chip design can facilitate this characterisation. We also consider how controlling the profile of the incident beam can affect the signal. We show that the efficacy of Raman spectroscopy in the liquid phase is not limited to particular materials by then looking at other liquid phase systems such as biological samples.
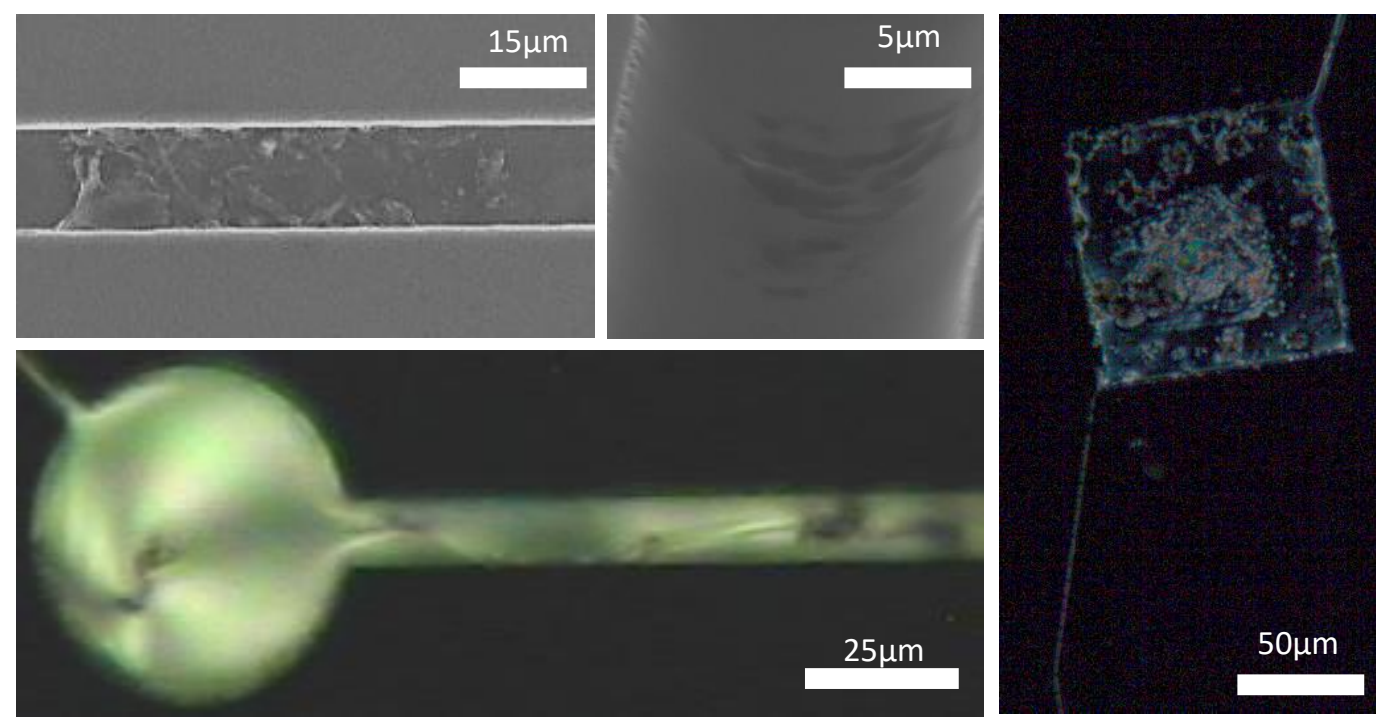

Figure 1: a) SEM image of graphene oxide flakes deposited in a microfluidic channel. b) SEM image of a graphene oxide flake dispersed in a nematic liquid crystal contained within a microfluidic channel with the meniscus clearly visible. c-d) Polarised light microscopy images of nematic liquid crystals with dispersed 2D materials contained within microfluidic features on-chip.
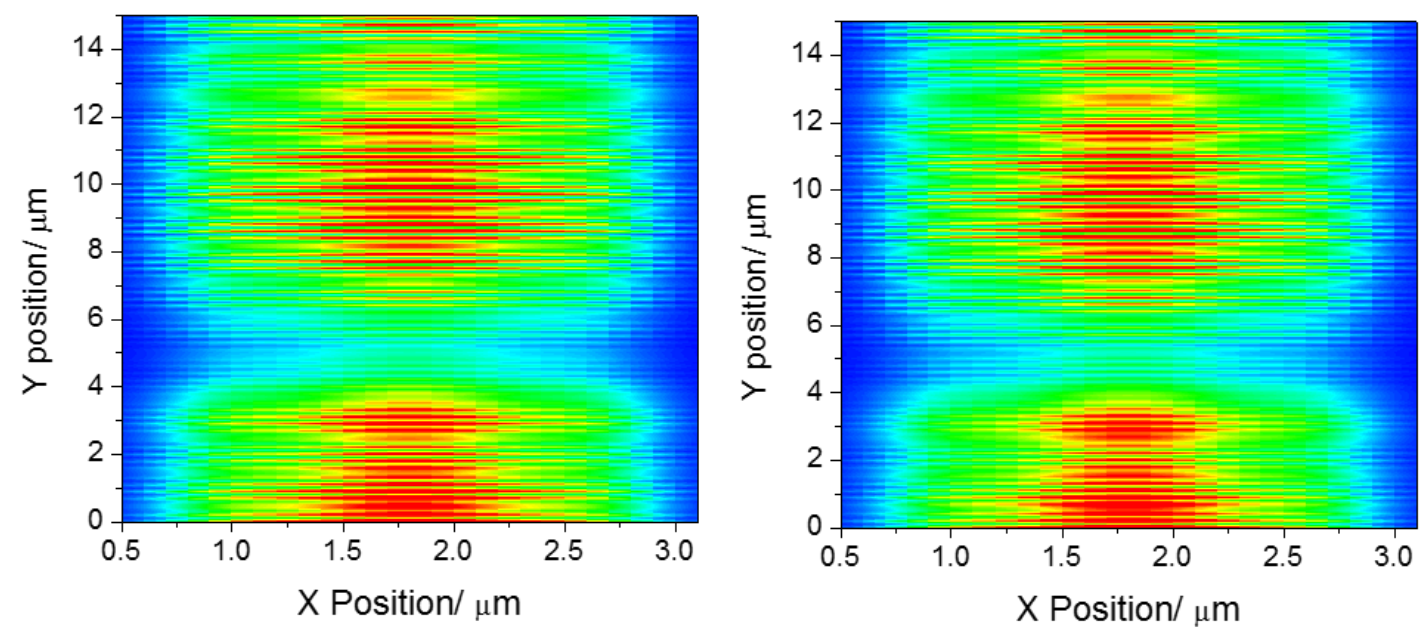

Figure 2: Predicted Raman intensity map for the molybdenum disulfide Raman band at a shifts of a) 384 $\mathrm{cm}^{-1}$ and b) $384 \mathrm{~cm}^{-1}$ with an excitation wavelength of $532 \mathrm{~nm}$, for a flake dispersed in a nematic liquid crystal contained within a $3.6 \times 15 \mu \mathrm{m}$ cavity.

[1] B. Hogan et al, 'Dynamic in-situ sensing of fluid-dispersed 2D materials integrated on microfluidic Si chip,' Scientific Reports 7, 42120 (2017), doi:10.1038/srep42120. 